\title{
Conceal Don't Feel: Study of Interpersonal Closeness between High School Males in California
}

\author{
Candy $\mathrm{Li}^{1}$ and Erica Suh" ${ }^{1 \#}$ \\ ${ }^{1}$ Maranatha High School, Pasadena, CA, USA \\ \#Advisor
}

\section{$\underline{\text { ABSTRACT }}$}

Recognizing the importance of genuine connection for human, this research dives into the study of interpersonal closeness between high school males in California. This paper includes a literature review with current scholarly work, consensus, and polls on interpersonal closeness and cultural views and effects through gender stereotypes. In the research design, a mixed method including causal-comparative research, content analysis, and coding was used. The main source of data was collected through interview responses. The responses were compared, coded, and analyzed. The conclusion that high school males in California have the capability of producing interpersonal closeness with each other was reached. Furthermore, differences were found in the ways female pairs and male pairs develop interpersonal closeness. Implication of encouraging interpersonal closeness within males were suggested.

\section{Introduction}

\author{
"Our souls \\ Will not be soothed \\ By what we achieve \\ How we look \\ Or all the hard work we do \\ Even if we manage to make all the money in the world \\ We'd be left feeling empty for something \\ Sour souls ache for community \\ Our deepest being craves one another \\ We need to be connected \\ To feel alive" \\ - Rupi Kaur
}

Humans are social creatures who crave genuine connection. When people open up, become vulnerable, and share parts of themselves with another, they include others in the self. Genuine connections can originate from feelings of interpersonal closeness that "occurs cognitively whenever one perceives an overlap between himself or herself and another person" (Aron et al., 1997). The key to a genuine connection that makes interpersonal relationship possible has been studied throughout history. Different paradigms, or models of widely accepted concepts and ideas, have been formed in an attempt to measure and produce closeness between people. Although studies of interpersonal relationships have been prominent in social sciences since the 19th, a 21st century male living up to the expectation of masculinity lacks the necessary education to understand and create a healthy affiliation based on the articulation of vulnerabilities. The modern idea of masculinity in America degrades signs of vulnerability, condemns display of personal feelings, and bars men from forming interpersonal relationships. A constant suppression of emotions results in unhealthy outcomes 
such as violence and aggression towards self and others. The reality is that humans, despite societal impacts, are still biologically social creatures and value connection with others, further demonstrating the importance of interpersonal closeness for all.

This research aims to answer the following questions: In a society where males are often taught to suppress their emotions, would high school males in California be able to develop interpersonal closeness with each other? If so, does the way high school males develop interpersonal closeness with each other differ from the way they develop interpersonal closeness with high school females and the way high school females develop interpersonal closeness with each other? If so, in what ways do they differ?

\section{Literature Review}

A 2017 study by Villanueva on interpersonal closeness, "Interpersonal Closeness, Self-Disclosure, and Attachment Styles of University Students in the Philippines," concluded that interpersonal intimacy and self-disclosure directly affect relationship satisfaction and commitment while attachment style does not. This demonstrates the importance of sharing and opening up to another person for the generation of closeness in return. However, as only Female-Male Pairs were used in the study, and the topic of male-male relations, serves as a gap in knowledge.

In a 1997 study by Aron et al, a paradigm was made to measure interpersonal closeness. "The Experimental Generation of Interpersonal Closeness: A Procedure and Some Preliminary Findings" set the foundation of future interpersonal closeness studies (this one included) by breaking down and studying the aspects that lead to temporary feelings of closeness, eliminating factors that do not, and creating a question-asking system for the generation of interpersonal closeness. In the study, it was concluded that disagreement on essential topics do not necessarily affect interpersonal closeness between two people, whether or not participants were made to expect mutual liking does not affect the outcome of the generated interpersonal closeness, making closeness an explicit task also served no significant difference to the outcome, introverts generally felt a little less closeness than that of extroverts, and that similarity and the expectation of attraction did not affect attraction or closeness. Those areas were not considered for this research because it has been concluded that those factors will not affect the outcome of closeness.

A gap within Aron et al.'s research, however, was the topic of gender. In the study, university students were grouped into Female Pairs and Female-Male Pairs that knew little about each other. However, no pairs of males used in the experiment due to the unbalanced ratio between females and males in the psychology class the study took place in, further proving the gap in knowledge in interpersonal relationships between males.

The psychology class used in the study is not a stand-alone case either. As of 2018, the ratio for the male to female graduate students in psychology is about 1:3 (Fowler et al., 2018). In fact, only $12 \%$ of men make up the personal care workers, $24 \%$ for health associate professionals, $32 \%$ for teaching professionals, and $34 \%$ for customer service clerks ("These Occupations," 2020). Now, what do these female-dominated occupations all have in common? They are all related to health care, interactions with others, and provision of support. In the 2019 US census, it was shown that about $75 \%$ of healthcare-related occupations are dominated by women (Day \& Christnacht, 2019). Like how many girls grow up believing they are not meant to be in STEM due to the lack of representation, with little representation of men in those fields, males grow up believing that they are not to show characteristics such as providing care, healthy interactions, and communication. The unbalanced workforce is just one example of how society infiltrates aggressive gender norms and expectations, further divert males from getting in touch with their feelings.

As individuals make up a society, what people believe about gender norms also carries significant weight in this discussion. A 2017 poll on what traits Americans valued and devalued in men and women was carried out. The results showed that "honesty/morality," "professional/financial success," "ambition/leadership," and "strength/roughness" were the most valued traits for men. In contrast, "physical attractiveness," "empathy/nurturing/kindness," "intelligence," and "honesty/morality" were the most valued traits for women (Parker et al., 2017). With strength and success so high on the list for men, it explains American culture's emphasis on the need for males to be strong and to be able to provide. 
Gender roles shape a person's identity consciously and subconsciously because it is ingrained in the social environment. Just as representation plays a role in shaping the environment a person will grow up in, how people are socialized and taught to behave is also an essential part of how people develop emotional responses, expressions, or the lack thereof. Backing this claim is Sandra Bem's Gender Schema Theory that explains how children develop sexdefined traits and behaviors from their environments. Biologically, men typically show higher blood pressure and cortisol responses to emotionally arousing stressors (especially achievement challenges) but are socialized to keep their emotional expression. (Stroud et al., 2002). In fact, a study conducted by Ross Buck in 1997 found that from the age of 4 to 6, while boys' emotion expression decreased, or have been dampened, girls' have not. Further backing up this study is a 2017 poll carried out by Walker et al. from Pew Research Center that found that while the words "compassionate," "caring," "kind," and "responsible" were considered positive traits for women, the terms were viewed more negatively as traits for men. In turn, these internal biases play a factor because when compared to their female counterparts, males demonstrate higher rates of antisocial behaviors, struggles with alcohol abuse which coincides and causes higher expressions of anger and lower expression of anxiety and sadness (Chaplin, 2008).

Although common for American males to have suppressed emotional expression, this does not mean males do not feel and cannot express their emotions. A 2013 meta-analysis by Chaplin and Aldao found that "gender differences in positive expressions in 0-17 year olds were strongest when with an unfamiliar adult and were not significant when with a parent, when alone, or when with a peer," showing that the more comfortable and familiar a child is with the person they are interacting with, the less prevalent will be their abeyance to gender stereotypes. As self-disclosure leads to familiarity and interpersonal closeness and familiarity will lead to more likeliness of abandoning societal gender stereotypes, including the expectations for males to close off emotions, hence, creating a positive cycle of the generation of interpersonal closeness. Because of this, it is hypothesized that though it may be challenging to open up, high school males in California do have the capability of producing interpersonal closeness with one another, but the way they produce interpersonal closeness may differ from the way they produce interpersonal closeness with females and the way females produce interpersonal closeness with each other.

\section{Research Design}

\section{Methodology}

The causal-comparative research method was chosen because it aims to identify causal relationships in variables that cannot be manipulated. For this research, the variable that could not be manipulated was the sex of the participants. This method was used to look at whether the sex of the participant pairs would affect their ability to develop interpersonal closeness with each other and to develop further understanding in interpersonal closeness between High School males in California.

Firstly, the experimental portion of this study was divided in a Control and Experimental Group. Within the Control Group, three Female Pairs of various initial closeness and three Female-Male Pairs of various initial closeness were used. The Experimental Group consisted of six Male Pairs with various initial closeness. The results of the Experimental Group were compared to the results of the Control Group to analyze the data. In the literature review, it was understood that Female Pairs and Female-Male Pairs have the capability of developing interpersonal closeness with each other, so the main purpose of the Control Group was to compare its results with the results of the Experimental Group. For the sake of limiting factors, only those who identify as males or females were used and studied, but for future implications, it would serve justice to study further into interpersonal closeness between those that do not fall under binary gender identities.

Various platforms (email blast and social media platforms that were not my own) were used for the recruitment of participants. For the sake of accessibility and eliminating excess variables only High Schoolers in California were used. An advantage of having participants be from California is that California is the most populous state with 
the most socioeconomic diversity, household diversity, and cultural diversity (Clarke, 2020). This ensures that the interviewees used are able to represent a variety of backgrounds.

Ranging from 16-17 years of age, the mean age of the participants was 16.25. Parental consent forms and individual consent forms were signed for the sake of ethical research. It was made known to participants they could withdraw from or withdraw their information from the research at any time during or after the interview.

Secondly, interviews were conducted. Due to the circumstances that the research was conducted amidst the outbreak of the COVID-19 pandemic, all interviews were held via Google Meet. Google Meet was chosen because at the time, it allowed free meetings with no time constraints. A limitation is that there were a few instances of internet problems. Silver-linings about holding the interviews online was that I was able to sit in on the interviews with my camera off (I was essentially a non-distracting black screen), my audio off, the participants were able to pin each other's videos to the screen, and the interviews were able to be recorded without having a camera blaring in the faces of the participants. To the best of our abilities, the participants and I used the tools and situation at hand (Google Meets) to create an environment that felt like it was just the two participants talking to each other for the most part. For the sake of easy access, the interviews were recorded with permission (via consent forms shown in the appendix) for it to be seen by only the researcher.

Before the interview, participants were asked to try their best to have access to a fully charged device with good internet service, minimal distractions, and privacy. In addition, they were asked to be in a place where they felt comfortable physically and emotionally and to have any accommodations (i.e., a cup of tea, a blanket, a pillow) that would maximize their physical and emotional comfort.

\section{Limitations}

The research was conducted to be as representative as possible, but I recognize that the six females and 18 male participants cannot not represent everyone in California. All participants were High Schooler from California because those were the group of people most readily available to the researcher, hence the specificity in the research question. Having participants only be from California may also be a limitation because the culture of California is different from the culture from other states. Therefore, the findings of this research only directly point to High Schoolers in California.

Another limitation is personal biases. Everyone has their own beliefs, but I tried to limit personal biases as much as possible by using statistics, coding, and data to back up the findings. The COVID-19 pandemic also caused a discrepancy in research in that for optional safety, interviews were conducted over Google Classroom opposed to face to face. This may have affected the way participants felt towards their partners, but given the circumstances, this was the only feasible way to hold interviews. Procedures during interviews, however, were held the same for all interviews to limit further possible discrepancies.

\section{Pre-Interview and Post-Interview Surveys}

Before and after each interview, participants were asked to complete a Pre-Interview Survey and a Post-Interview Survey. The purpose of the surveys was to gather information about how participants felt towards their partners before and after the interviews. The information gathered were then compared to make conclusions about interpersonal closeness between high school males in California. The Pre-Interview Survey (Table 1) included only and IOS Scale, and the Post-Interview Survey included follow up questions that prompted free response answers (Table 2). The IOS Scale (Inclusion of Others in the Self Scale), crafted by Aron et. al, with the purpose of measuring how close one person feels about another, was used with the permission from Dr. Aron. A visualization of the IOS Scale is presented in Figure 1. 


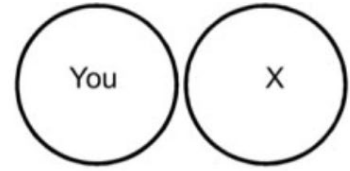

1

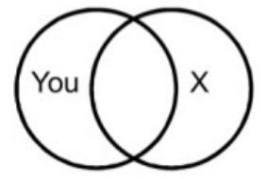

4

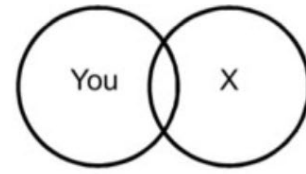

2

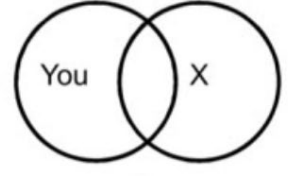

3

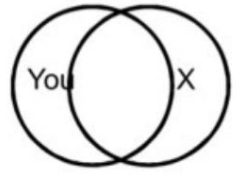

5

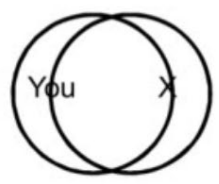

6

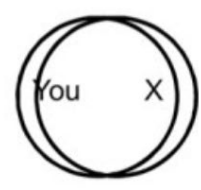

7

Figure 1. Inclusion of Others in the Self Scale. The IOS Scale shows numbered diagrams from 1 to 7 (1 being the least close, 7 being the closest). Participants were asked to choose the number they felt most associated with how they felt towards their partner before and after their interview.

The IOS Scale was chosen for its simplicity and neutrality, allowing people to use their own perception on their feelings towards their partner. It was included in both the Pre-Interview and Post-Interview surveys to compare if individuals had any increased feelings of closeness. The IOS Scale in the Pre-Interview Survey determined the initial closeness number participants felt towards their partners and the Control and Experimental Groups were both further divided into categories based on that. The reasoning behind this step is that in the matching of pairs of partners for the interviews, evenly distributed initial feelings of closeness was sought out to see if differences in initial feelings of closeness would be significant in developing interpersonal closeness. Numbers 1-3, 3-5, and 5-7 make up the three Initial Closeness Categories within the groups. Numbers within the categories slightly overlap because it is important to recognize that people interpret the IOS scale differently, one number does not neatly translate to a specific feeling, and two people that feel the same amount of closeness towards another may still choose different numbers.

After the completion of the Pre-Interview Survey (seen in table 1), with permission from the creator, Dr. Aron, the 36 Questions meant to help generate interpersonal closeness, guided the conversation between participants during the interviews. See Appendix for full question set. Each pair had the choice of viewing those questions on a website (created by the researcher for this research) and having me manually put the questions one by one in the chat as they progressed. This choice was given for the reason that participants had access to different devices and while it was easier for some to use the website (see appendix), others found it easier to have questions put in the chat. For the pairs that used the website, they were asked to use a split screen so they could see the questions and their partners simultaneously. The inconsistency in how the questions were accessed may serve as a limitation, but for the sake of accommodating differences in technology, the call for a choice was made. With the questions divided into three sets of 12 questions that gradually became more personal, participants took turns asking the questions, both participants' responses to each question, and participants all went through the questions at their own pace. After the questions were asked and answered, participants were given the choice to fill out their Post-Interview Survey in the same room with their partners or in a separate breakout room. The Post-Interview Survey included an IOS Scale, direction for participants to identify if any of the 36 Questions made them feel closer to their partners, to elaborate how the identified questions made them feel, and to identify and to explain their choice regarding the breakout room. The Post-Interview Survey questions are presented in Table 2. 
Table 1. Pre-Interview Survey Question

\begin{tabular}{l} 
Question \\
If you do feel you've gotten closer to your interview partner during the process, \\
please attempt to identify when (during which question(s)) you started to feel \\
closer and explain in detail how you felt. \\
\hline IOS Scale
\end{tabular}

Table 2. Post-Interview Survey Questions

\begin{tabular}{|l|l|l|}
\hline Question 1 & Question 2 & Question 3 \\
\hline $\begin{array}{l}\text { If you do feel you've gotten closer to } \\
\text { your interview partner during the } \\
\text { process, please attempt to identify } \\
\text { when (during which question(s)) } \\
\begin{array}{l}\text { you started to feel closer and explain } \\
\text { in detail how you felt. }\end{array}\end{array}$ & $\begin{array}{l}\text { your interview partner during the } \\
\text { process, please attempt to identify } \\
\text { when (during which question(s)) } \\
\text { you started to feel closer and explain } \\
\text { in detail how you felt. }\end{array}$ & $\begin{array}{l}\text { Did you choose to fill out this survey } \\
\text { in a breakout room by yourself or in } \\
\text { the same room as your partner? Why } \\
\text { did you make this choice? Please ex- } \\
\text { plain in full detail. }\end{array}$ \\
\hline
\end{tabular}

Thirdly, after interviews were conducted, surveys were filled out, and Initial Closeness Categories were made, through mixed methods, data was collected from the surveys. The surveys, instead of the actual interview, was where data was collected from because one, how and what the interview made participants feel was what the concern of the research, not the specific things said during interviews, and two, the interviews were solely confidential, and the privacy of participants were respected. Qualitative information was gathered from the surveys and then analyzed through coding and content analysis.

Coding, the act of defining data through grouping and identifying relations between concepts and words, was chosen for this research because it is a simple way of compressing and comprehending the Post-Interview Surveys responses and it paved the way for content analysis that is crucial to the findings. The Post-Interview Surveys responses of participants are categorized into themes, the themes are then studied for possible relations, and the findings are further developed in content analysis. One reason it was chosen was because content analysis is usually used for social lenses (as this is a sociological and psychological research). Another reason is that because content analysis is used to quantify and analyze the meanings and relationships of words and concepts within writers and their texts. Through coding and content analysis, themes, ideas, and quantitative data are found and compared.

\section{Findings}

In looking at whether there were changes between the Closeness Number in the Pre-Interview IOS Scale and the PostInterview IOS for each participant, it was determined if those individuals felt increased feelings of closeness towards their partner. The percentage of increased feelings of closeness within each Initial Closeness Category was found 
through solving for the mean percent change. The key portion of this study was to see if there were positive percentages greater than zero within the Male Pairs, because that would indicate increased feelings of closeness. A visualization of the data mentioned is presented in Table 3 and Table 4.

Table 3. Percentage of Increased Feelings of Closeness in the Control Group

\begin{tabular}{|l|l|}
\hline Initial Closeness Categories & \% Increase in Feelings of Closeness \\
\hline Female Pairs (1-3) & $229.20 \%$ \\
\hline Female Pairs (3-5) & $66.70 \%$ \\
\hline Female Pairs (5-7) & $0 \%$ \\
\hline Cross Sex Pairs (1-3) & $250 \%$ \\
\hline Cross Sex Pairs (3-5) & $22.50 \%$ \\
\hline Cross Sex Pairs (5-7) & $20 \%$ \\
\hline
\end{tabular}

Table 4. Percentage of Increased Feelings of Closeness in the Experimental Group

\begin{tabular}{|l|l|}
\hline Initial Closeness Categories & \% Increase in Feelings of Closeness \\
\hline Male Pairs (1-3) & $283.40 \%$ \\
\hline Male Pairs (3-5) & $60.10 \%$ \\
\hline Male Pairs (5-7) & $8.60 \%$ \\
\hline
\end{tabular}

For the portion of the Post-Interview Survey that asked participants to identify the question(s), if any, that made them feel closer to their partner, the most chosen questions within the Control and Experimental Group were coded. The coding mentioned is presented in Table 5.

Table 5. No Theme(s) Were Unique to Male Pairs

\begin{tabular}{|l|l|l|l|l|}
\hline Groups & Themes & \\
\hline Female Pairs & Interactive & Compliments & Elaboration & Similarity \\
\hline Cross Sex Pairs & Interactive & Compliments & Elaboration & \\
\hline Male Pairs & Interactive & Compliments & Elaboration & \\
\hline
\end{tabular}

Note. This table shows coding of the most chosen 36 Questions that made participants feel closer to their partner.

In the part of the Post-Interview Survey that asked participants to elaborate on why they felt closer to their partner, if they had at all, the responses were coded into themes. The coding mentioned is presented in Table 6 .

Table 6. Trust is a Theme Found Unique to Male Pairs

\begin{tabular}{|c|c|c|c|c|}
\hline Groups & \multicolumn{4}{|l|}{ Themes } \\
\hline Female Pairs & $\begin{array}{l}\text { closer due to know- } \\
\text { ing more about each } \\
\text { other. }\end{array}$ & & & \\
\hline Cross Sex Pairs & $\begin{array}{l}\text { closer due to know- } \\
\text { ing more about each } \\
\text { other. }\end{array}$ & related to each other & $\begin{array}{l}\text { felt potential growth } \\
\text { in their relationship }\end{array}$ & \\
\hline Male Pairs & $\begin{array}{l}\text { closer due to know- } \\
\text { ing more about each } \\
\text { other. }\end{array}$ & related to each other & $\begin{array}{l}\text { felt potential growth } \\
\text { in their relationship }\end{array}$ & $\begin{array}{l}\text { trusted their partner } \\
\text { more }\end{array}$ \\
\hline
\end{tabular}

Note. This table shows the coding of responses to the question on why participants felt closer to their partner, if they did feel closer at all. 
For the portion of the Post-Interview Survey that asked participants to explain their choice regarding the breakout room, the responses were coded into themes. Every participant decided against going into their own breakout rooms and their reasoning was coded into themes. The coding mentioned is presented in Table 7.

Table 7. Trust is a Theme Found Unique to Male Pairs Again

\begin{tabular}{|c|c|c|c|c|c|}
\hline Groups & \multicolumn{5}{|l|}{ Themes } \\
\hline Female Pairs & nothing to hide & $\begin{array}{l}\text { felt comfortable } \\
\text { with partner }\end{array}$ & $\begin{array}{l}\text { wanted to stay } \\
\text { and talk with } \\
\text { partner }\end{array}$ & & \\
\hline Cross Sex Pairs & nothing to hide & $\begin{array}{l}\text { felt comfortable } \\
\text { with partner }\end{array}$ & & practical to stay & \\
\hline Male Pairs & nothing to hide & $\begin{array}{l}\text { felt comfortable } \\
\text { with partner }\end{array}$ & $\begin{array}{l}\text { wanted to stay } \\
\text { and talk with } \\
\text { partner }\end{array}$ & practical to stay & $\begin{array}{l}\text { trusted their } \\
\text { partner }\end{array}$ \\
\hline
\end{tabular}

Note. This table shows coding for responses to the question why participants decided against being put into separate breakout rooms.

\section{Discussion and Analysis}

\section{Percentage of Increased Feelings of Closeness}

As shown in Table 3 and 4, the study of the percentages of increased feelings of closeness looked at whether or not there would be an increase in closeness for the male pairs. The data showed that not only did the Male Pairs in the Experimental Group show significant increase in closeness, the extent of the increase in the 1-3 Category, and 3-5 Category even succeeded that of the Control Group. The finding concludes that High School males in California have capability of opening up to each other, generating closeness with each other, and developing interpersonal closeness with each other despite societal pressures. This also concludes that the first portion of the initial hypothesis is correct.

\section{Questions that made Participants Feel Closer}

Found in Table 5, the coding of the questions that made participants feel closer to their partners shows the themes that while questions that led participants to identify similarities with their partner was a theme found within the responses of the Female Pairs, everything else remained the same for the Control and the Experimental Groups. Questions that were interactive, had to do with giving and receiving compliments, and had participants elaborate on their responses were prevalent within all groups. This finding shows that identifying similarities may lead to females feeling closer to each other but does not show any unique factors that made the Male Pairs feel closer to each other. For future implications, it is recommended that further research be conducted to examine how identifying similarities affect feelings of closeness females.

\section{Why Participants Felt Closer to Their Partner}

Themes found in the responses to why participants felt closer to their partners was that across the board, the Female Pairs and Cross-Sex Pairs of the Control Group and the Male Pairs of the Experimental Group all felt closer because they got to know more about their partner, they related to their partner, and they saw potential growth in their relationship. The one theme that was only found in the Male Pairs, however, was that trusting their partners more made the participants feel closer. 


\section{Breakout Rooms?}

Because interviews were conducted online through Google Meets, participants had the option to be put into a separate breakout room to fill out the last survey upon completing the interview. Every single participant decided not to be put into their own breakout rooms. For the Female Pairs, the reason behind this choice was that they felt like they had nothing to hide from their partners, they felt comfortable with their partner, and they wanted to stay and talk with their partners. The Cross-Sex Pairs stated that they did not feel the need to go into a separate room, it felt practical to stay, they had nothing to hide, and they felt comfortable with their partners. The Male Pairs' reasons overlapped with the reasons of the Control Groups meaning that they also felt it was practical to stay, they had nothing to hide, they felt comfortable with their partners, and they wanted to stay and talk. But the one thing that differed was that another theme found within the Male Pairs was that they decided to stay because they trusted their partners. The theme of trust was found once again in the Male Pairs; This proves that the final portion of the hypothesis is correct because it concludes that the way the male pairs developed interpersonal closeness with each other differed from the pairs of the Control Group in the sense that while the concept of trust was found as one of the themes that led the Male Pairs to closeness, it was not found in the Female Pairs or Cross-Sex Pairs. This may mean that trust plays a bigger role leading to interpersonal closeness in male pairs than other groups.

\section{Conclusion}

\section{Practical Implications}

Having filled the gap in pre-existing research about interpersonal closeness between High School males and concluding that High School males have the capability to develop interpersonal closeness with each other and that trust plays a factor in the development, teenage males should be encouraged to be more vulnerable. The impact of this research shows that in being vulnerable, interpersonal closeness is formed and this closeness leads to a sense of community and intimacy that betters mental health of individuals. The toxic culture that teaches males to conceal harms everyone because those around the unfeeling males with deteriorating mental health are affected as well. As mentioned in the literature review, emotional suppression often leads to unhealthy aggression, anger, and even substance abuse (Chaplin, 2008), reversing this emotional suppression as a community would be a good step towards creating healthier relationships and emotional regulation skills in males. So, in eradicating the stigma around vulnerability in males will benefit not only high school males, but society as a whole.

But because society will not change itself, individuals that make up society could change society, its gender stereotypes, stigmas, and work to better support this vulnerability and emotional expression within males. "Parents should review their behavior to make sure they're not doing or saying anything that feeds into something harmful" (Bosse, 2017) This review of behavior is not limited to parent's either. It is true that parents play a big role in shaping individuals, but everyone has the capability of greatly impacting and influencing those around them. Applauding self and emotional expression, checking one's own internal biases, reminding others of theirs, and questioning the idea that perpetuating gender stereotypes and that lead to certain biases are ways individuals can help encourage vulnerability in males.

Males should be given safe spaces to have more conversations about their feelings and they should be encouraged to take off the "mask of masculinity" (Pollack, 1999). Because what is behind that mask? What is behind that masculinity? Males should be encouraged to pursue that question and seek for who they are within. It takes courage to be who you are, and it takes courage to be vulnerable. Instead of dampening that vulnerability and expression, as a society, we should support and nurture it so males would not be caught up in an aggressive and angry wall with pent up sadness and anxiety bottled up inside. 
Ridding the stigma around males being vulnerable can help males feel more confident expressing their feelings. To rid the stigma, the issue needs to be talked about. Institutions, peers, parents, and males themselves can all contribute to the destigmatizing males showing emotions. Phrases such as "boys don't cry," "man up," and "stop acting like a girl" and assigning certain colors and actions to a specific sex has a lot more impact than it seems (Bosse, 2017) for it reinforces a very rigid idea of masculinity and femininity. Challenging these stereotypes can play a part in ridding the stigma for boys who do cry, there is no one way to be a man, liking pink, and working in health care (a female dominant field) does not take away one's masculinity. To advocate against those rigid stereotypes, it is helpful to recognize one's own bias.

Personal biases exist within all humans, and everyone has different beliefs. While it is normal to have biases and different beliefs, understanding why those internal biases exist can help people determine if they actually agree with it or just have been conditioned to. In order to understand, one should question their biases and stereotypes that may have contributed to it. Gender stereotypes of all sorts, not just for males, are of the topics that should be questioned. They should be questioned because stereotypes of all genders are linked to each other and indirectly contribute to stereotypes regarding different genders. For example, it may be socially and culturally acceptable and expected for females to wear makeup and the color pink. But females are not the only group that can wear makeup and the color pink, and females do not have to wear makeup and the color pink. In questioning these stereotypes directed towards females, it can make it easier for males and non-females to express themselves with makeup and pink. Like how opening up to another person is a form of self-disclosure, wearing what one feels good in is another form of selfdisclosure. Therefore, expressing oneself in the way they wish will also lead to vulnerability, and that vulnerability is generated with the questioning of expectations. This goes to show that encouraging vulnerability in males does not always directly have to do with males and that vulnerability can be generated in different ways other than just in conversations.

Those with more power, resources, and audience such as institutions, corporations, and facilities that comes in regular contact with males have a greater platform that can promote the importance vulnerability on a bigger scale. As most of the participants expressed positive feelings towards the interview for it helped guide them through conversations that made them feel closer to their partners those institutions, corporations, and facilities can host similar exercises to teach people about internal biases they may hold, help them identify ways they may be intentionally or unintentionally suppressing feelings, help people open up to each other, and start meaningful conversations about mental health, stigma, vulnerability in males and everyone else too.

\section{Future Implications}

For future research, how demographics such as age, gender identity (those who do not identify as female or male), geography, and culture may influence interpersonal closeness should be studied.

An interesting finding in the Post-Interview Surveys is that while all groups showed some increase in closeness, the Male-Male groups were the only ones that mentioned trust as a propeller for the increase in closeness. It may be worthwhile to use a larger sample group to look into trust in High School males and males in general, whether it just a coincidence of none of the Female and Cross-Sex Pairs brought up trust, how people of different gender identities may view trust differently, what roles trust plays in relationships for different gender identities.

It is also recommended that further research study how identifying similarities affect feelings of closeness within females because that was also a theme found to be unique to causing increased feelings of closeness within Female Pairs. This study did not focus on interpersonal closeness within females, but further understanding about interpersonal closeness within other gender identities (in this case females), is also important in contributing to knowledge of interpersonal closeness. 


\section{Closing -- An Original Poem}

Boys, you are not heartless

And your suppression of expression

Definitely is not harmless

Our deranged definition

Of masculinity

Is what led to repressed vulnerability

Fall not under the impression

That aggression and depression is the only thing in store

For let us lead the transgression

And open a door

To raw, true emotions

And to discussions and questions

It'll be better for everyone for sure

\section{Acknowledgements}

I want to express my deepest gratitude to Mr. Fenderson from Maranatha High School that supported me through the research process. Our numerous discussions inside and outside the classroom lead this project to achieve what it otherwise would not have. Secondly, I would like to thank my expert adviser Erica Suh, and other teachers from Maranatha High School for that helped me gain more knowledge about the field of psychology and provided me with advice about my research. Thirdly, I am incredibly grateful for every individual that participated in my research interviews, for without them, this project would not have been possible. In addition, gratification is due to Dr. Aron for allowing me to use the 36 Questions and IOS Scale. Last but certainly not least, I appreciate everyone that has been of support to me during the intensive yet exciting journey of creating and completing this research project and to everyone that has expressed interest, believed in me and pushed me to become a better researcher.

\section{References}

Aron, A., Aron, E. N., \& Smollan, D. (1992). Inclusion of Other in the Self Scale and the structure of interpersonal closeness. Journal of Personality and Social Psychology, 63(4), 596-612. https://doi.org/10.1037/0022$\underline{3514.63 .4 .596}$

Aron, A., Melinat, E., Aron, E. N., Vallone, R. D., \& Bator, R. J. (1997, April 1). The Experimental Generation of Interpersonal Closeness: A Procedure and Some Preliminary Findings. SAGE Journals . https://doi.org/10.1177/0146167297234003.

Bialik, K., Kessel, P. van, \& Walker, K. (2021, February 9). How Americans describe what society values in men and women. Pew Research Center's Social \& Demographic Trends Project. https://www.pewresearch.org/social-trends/interactives/strong-men-caring-women/. 
Bosse, J. (2017, June 22). How 'Man Up' And Other Stereotypical Sayings Hurt Boys. HuffPost. https://www.huffpost.com/entry/how-man-up-and-other-stereotypical-sayings-hurtboys b 594ac2b4e4b092ed90588b28.

Buck, R. (1976. 2nd Ed. 1988). Human Motivation and Emotion. New York: Wiley.

Chaplin, T. M., \& Aldao, A. (2013, July 1). Gender Differences in Emotion Expression in Children: A MetaAnalytic Review. National Center for Biotechnology Information. https://www.ncbi.nlm.nih.gov/pmc/articles/PMC3597769/.

Chaplin, T. M. (2015, June 16). Gender and Emotion Expression: A Developmental Contextual Perspective. National Center for Biotechnology Information. https://www.ncbi.nlm.nih.gov/pmc/articles/PMC4469291/\#R17.

Day, J. C., \& Christnacht, C. (2020, August 18). Your Health Care Is in Women's Hands. The United States Census Bureau. https://www.census.gov/library/stories/2019/08/your-health-care-in-womenshands.html\#: : :text=Women \%20Hold \%2076\%25\%20of\%20All,Gaining\%20in\%20Higher\%2DPaying\%20 Occupations.

Fowler, G., Cope, C., Michalski, D., Christidis, P., Lin, L., \& Jessica. (2018, December 28). Women outnumber men in psychology graduate programs. American Psychological Association .

Parker, K., Horowitz, J. M., \& Stepler, R. (2020, August 6). 2. Americans see different expectations for men and women. Pew Research Center's Social \& Demographic Trends Project. https://www.pewresearch.org/social-trends/2017/12/05/americans-see-different-expectations-for-men-andwomen/.

Pollack, W. (1999). Real boys: rescuing our sons from the myths of boyhood. Scribe Publications.

Stroud, L. R., Salovey, P., \& Epel, E. S. (2002, August 15). Sex differences in stress responses: social rejection versus achievement stress. National Center for Biotechnology Information. https://pubmed.ncbi.nlm.nih.gov/12208639/.

These occupations are dominated by women. ILOSTAT. (2020, July 17). https://ilostat.ilo.org/these-occupationsare-dominated-bywomen/\#: :text=By $\% 20$ far $\% 20$ the $\% 20$ most $\% 20$ female,are $\% 20$ mostly $\% 20$ filled $\% 20$ by $\% 20$ women.

U.S. News \& World Report. (2020, September 10). The Most Diverse States in the U.S. U.S. News \& World Report. https://www.usnews.com/news/best-states/articles/2020-09-10/california-is-the-most-diverse-state-in-theus\#: : text=California $\% 20 I s \% 20$ the $\% 20$ Most $\% 20$ Diverse $\% 20$ State $\% 2$ C $\% 20$ Report $\% 20$ Says,facets $\% 20$ con sidered $\% 20$ in $\% 20$ this $\% 20$ ranking.

Villanueva, L. S. (2017, February). INTERPERSONAL CLOSENESS, SELF-DISCLOSURE, AND ATTACHMENT STYLES OF UNIVERSITY STUDENTS IN THE PHILIPPINES. Jesco. 